\title{
EXISTENCE AND NONEXISTENCE OF POSITIVE SOLUTIONS TO A RIGHT-FOCAL BOUNDARY VALUE PROBLEM ON TIME SCALES
}

\author{
ILKAY YASLAN KARACA
}

Received 10 October 2005; Revised 19 January 2006; Accepted 30 January 2006

We are concerned with proving the existence of one or more than one positive solution of an $n$-point right-focal boundary value problem for the nonlinear dynamic equation $(-1)^{n-1} x^{\Delta^{n}}(t)=\lambda r(t) f\left(t, x^{\sigma}(t)\right)$. We will also obtain criteria which lead to nonexistence of positive solutions. Here the independent variable $t$ is in a time scale. We will use fixed point theorems for operators on a Banach space.

Copyright (c) 2006 Ilkay Yaslan Karaca. This is an open access article distributed under the Creative Commons Attribution License, which permits unrestricted use, distribution, and reproduction in any medium, provided the original work is properly cited.

\section{Introduction}

Motivated by the work of Anderson [3] on discrete third-order three-point right-focal boundary value problems, in this paper we will study an $n$ th-order $n$-point right-focal boundary problem on time scales. This paper also gives nonexistence and multiplicity results for positive solutions to the time scale boundary value problem

$$
\begin{aligned}
(-1)^{n-1} x^{\Delta^{n}}(t) & =\lambda r(t) f\left(t, x^{\sigma}(t)\right) \quad \forall t \in\left[t_{1}, \rho\left(t_{n}\right)\right], \\
x\left(t_{1}\right) & =x^{\Delta}\left(t_{2}\right)=\cdots=x^{\Delta^{n-1}}\left(t_{n}\right)=0
\end{aligned}
$$

where $n \geq 2, t_{1}<t_{2}<\cdots<t_{n-1}<t_{n}, \lambda$ is a real parameter, and $x=x(t)$ is a desired solution. The arguments are similar to those used in $[9,13]$.

In the third section we obtain multiplicity results for this problem with $\lambda=1$. In the fourth section existence, nonexistence, and multiplicity results are given for the eigenvalue problem.

To understand this so-called dynamic equation (1.1) on a time scale $\mathbf{T}$, we need some preliminary definitions.

Definition 1.1. Let $\mathbf{T}$ be a nonempty closed subset of $\mathbb{R}$ and define the forward jump operator $\sigma(t)$ at $t$ for $t<\sup \mathbf{T}$ by

$$
\sigma(t):=\inf \{s>t: s \in \mathbf{T}\}
$$

Hindawi Publishing Corporation Advances in Difference Equations Volume 2006, Article ID 43039, Pages 1-13

DOI 10.1155/ADE/2006/43039 
2 A right-focal boundary value problem on time scales

and the backward jump operator $\rho(t)$ at $t$ for $t>\inf \mathbf{T}$ by

$$
\rho(t):=\sup \{s<t: s \in \mathbf{T}\}
$$

for all $t \in \mathbf{T}$.

We assume throughout that $\mathbf{T}$ has the topology that it inherits from the standard topology on the real numbers $\mathbb{R}$. If $\sigma(t)>t$, we say $t$ is right scattered, while if $\rho(t)<t$, we say $t$ is left scattered. If $\sigma(t)=t$, we say $t$ is right dense, while if $\rho(t)=t$, we say $t$ is left dense.

We assume $\sigma^{0}(t)=t$, and for any integer $n>0$, we have

$$
\sigma^{n}(t):=\sigma\left(\sigma^{n-1}(t)\right)
$$

Throughout this paper we make the blanket assumption that $a \leq b$ are points in $\mathbf{T}$.

Definition 1.2. Define the interval in $\mathbf{T}$ :

$$
[a, b]:=\{t \in \mathrm{T} \text { such that } a \leq t \leq b\} .
$$

Other types of intervals are defined similarly.

We are concerned with calculus on time scales which is a unified approach to continuous and discrete calculus. In $[4,11]$, Aulbach and Hilger have initiated the development of this calculus. Since then, efforts have been made in the context of time scales, in establishing that some results for boundary value problems for ordinary differential equations and their discrete analogues are special cases of more general results on time scales; for a wide variety of problems addressed, see many references $[1,5,6,8-10,14]$.

Definition 1.3. Assume $x: \mathbf{T} \rightarrow \mathbb{R}$ and fix $t \in \mathbf{T}$ such that $t<\sup \mathbf{T}$, then $x^{\Delta}(t)$ is defined to be the number (provided it exists) with the property that, given any $\epsilon>0$, there is a neighborhood $U$ of $t$ such that

$$
\left|[x(\sigma(t))-x(s)]-x^{\Delta}(t)[\sigma(t)-s]\right|<\epsilon|\sigma(t)-s|
$$

for all $s \in U \cdot x^{\Delta}(t)$ is called the delta derivative of $x$ at $t$.

It can be shown that if $x: \mathbf{T} \rightarrow \mathbb{R}$ is continuous at $t \in \mathbf{T}, t<\sup \mathbf{T}$, and $t$ is right scattered, then

$$
x^{\Delta}(t)=\frac{x^{\sigma}(t)-x(t)}{\sigma(t)-t} .
$$

Note, if $\mathbf{T}=\mathbb{Z}$, where $\mathbb{Z}$ is the set of integers, then

$$
x^{\Delta}(t)=\Delta x(t):=x(t+1)-x(t)
$$

Moreover, if $\mathbf{T}=\mathbb{R}$, then

$$
x^{\Delta}(t)=x^{\prime}(t) .
$$


Finally, for $n \geq 1$, define

$$
x^{\Delta^{n}}(t):=\left\{x^{\Delta}(t)\right\}^{\Delta^{n-1}}
$$

assuming $x^{\Delta^{0}}(t)=x(t)$.

Definition 1.4. If $F^{\Delta}(t)=f(t)$, then define the integral of $f$ by

$$
\int_{a}^{t} f(\tau) \Delta \tau:=F(t)-F(a)
$$

Note that in the case $\mathbf{T}=\mathbb{R}$ we have

$$
\int_{a}^{b} f(t) \Delta t=\int_{a}^{b} f(t) d t
$$

and in the case $\mathbf{T}=\mathbb{Z}$ we have

$$
\int_{a}^{b} f(t) \Delta t=\sum_{k=a}^{b-1} f(k),
$$

where $a, b \in \mathbf{T}$ with $a \leq b$.

\section{Preliminaries}

As in [2], we introduce the Taylor polynomials $h_{j}: \mathbf{T}^{2} \rightarrow \mathbb{R}, j \in \mathbf{N}_{0}$, recursively defined as follows:

$$
\begin{aligned}
h_{0}(t, s) & =1 \quad \forall s, t \in \mathbf{T}, \\
h_{j+1}(t, s) & =\int_{s}^{t} h_{j}(\tau, s) \Delta \tau \quad \forall s, t \in \mathbf{T} .
\end{aligned}
$$

For integers $n \geq 2$ and for $i=1,2, \ldots, n-1$, define

$$
u_{n, i}(t, s) \equiv u_{n, i}\left(t, s: t_{1}, t_{2}, \ldots, t_{n}\right),
$$

with $t, s, t_{j} \in \mathbf{T}$ for $1 \leq j \leq n$, as follows:

$$
u_{n, i}(t, s):=(-1)^{n+1}\left|\begin{array}{ccccc}
0 & h_{1}\left(t, t_{1}\right) & h_{2}\left(t, t_{1}\right) & \ldots & h_{n-1}\left(t, t_{1}\right) \\
c_{2}(s, i) & 1 & h_{1}\left(t_{2}, t_{1}\right) & \ldots & h_{n-2}\left(t_{2}, t_{1}\right) \\
c_{3}(s, i) & 0 & 1 & \ldots & h_{n-3}\left(t_{3}, t_{1}\right) \\
\vdots & \vdots & \vdots & \ldots & \vdots \\
c_{n-1}(s, i) & 0 & 0 & \ldots & h_{1}\left(t_{n-1}, t_{1}\right) \\
1 & 0 & 0 & \ldots & 1
\end{array}\right|
$$

where

$$
c_{j}(s, i):=H(j-1-i) h_{n-j}\left(t_{j}, \sigma(s)\right),
$$


4 A right-focal boundary value problem on time scales

for $j=2,3, \ldots, n-1$ and $i=1,2, \ldots, n-1$. Here

$$
H(x)= \begin{cases}0 & \text { if } x<0 \\ 1 & \text { if } x \geq 0\end{cases}
$$

is the usual Heaviside function, and $h_{j}(t, s)$ is as defined in (2.2). In addition, define

$$
v_{n, i}(t, s):=u_{n, i}(t, s)+(-1)^{n-1} h_{n-1}(t, \sigma(s)),
$$

for integers $n \geq 2$ and for $i=1,2, \ldots, n-1$.

Theorem 2.1 [2]. For $u_{n, i}(t, s)$ as in (2.4) and $v_{n, i}(t, s)$ as in (2.7),

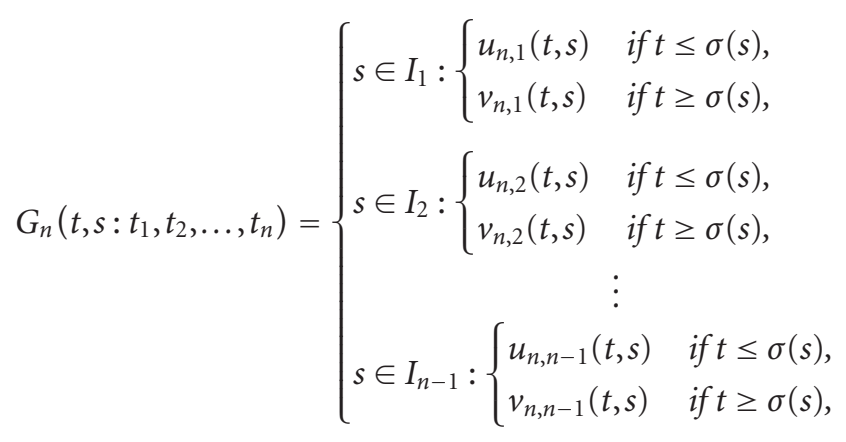

where $I_{1}=\left[t_{1}, \rho\left(t_{2}\right)\right]$, and $I_{i}=\left[\rho\left(t_{i}\right), \rho\left(t_{i+1}\right)\right]$ for $i=1,2, \ldots, n-1$, is Green's function for the homogeneous problem $(-1)^{n-1} x^{\Delta^{n-1}}(t)=0$ satisfying the boundary conditions (1.2).

Lemma $2.2[2]$. For $s \in\left[t_{1}, \rho\left(t_{2}\right)\right]$ and $n \geq 2$,

$$
G_{n}\left(t, s: t_{1}, t_{2}, \ldots, t_{n}\right) \begin{cases}<0 & \text { if } t \in\left(-\infty, t_{1}\right), \\ >0 & \text { if } t \in\left(t_{1}, \sigma^{n-1}\left(t_{n}\right)\right] .\end{cases}
$$

Theorem 2.3 [2]. Let $u_{n, i}(t, s)$ and $v_{n, i}(t, s)$ be given as in (2.4) and (2.7), respectively. Assume for $n \geq 4$ that

$$
v_{n-i, j-i+1}\left(\sigma^{n-i}\left(t_{n}\right), \sigma\left(s_{j}\right): t_{i}, t_{i+1}, \ldots, t_{n-1}\right)>0
$$

for $j \in\{2,3, \ldots, n-2\}$ and $i=j-1, j-2, \ldots, 1$, and for $s_{j} \in\left[\rho\left(t_{j}\right), \rho\left(t_{j+1}\right)\right]$. Then

$$
G_{n}\left(t, s: t_{1}, t_{2}, \ldots, t_{n}\right) \begin{cases}<0 & \text { if } t \in\left(-\infty, t_{1}\right), \\ >0 & \text { if } t \in\left(t_{1}, \sigma^{n-1}\left(t_{n}\right)\right]\end{cases}
$$


for $s \in\left[t_{1}, \rho\left(t_{n}\right)\right]$ if $n$ is even, or for $s \in\left[t_{1}, \rho\left(t_{n-1}\right)\right]$ if $n$ is odd. For odd $n \geq 3$, the additional assumption

$$
u_{n, n-1}\left(\sigma^{n-1}\left(t_{n}\right)\right)+(-1)^{n-1} h_{n-1}\left(\sigma^{n-1}\left(t_{n}\right), t_{n}\right)>0
$$

yields (2.11) for $s \in\left[\rho\left(t_{n-1}\right), \rho\left(t_{n}\right)\right]$ as well.

Throughout this paper, we assume that the time scale $\mathbf{T}$ is such that $\sigma(s)$ is delta differentiable for all $s \in \mathbf{T}, t_{1}$ is right-scattered, and hypotheses of Theorem 2.3 hold.

Furthermore, we have the following assumptions.

(H1) $r(s)$ is a nonnegative continuous function defined on $\left[t_{1}, \rho\left(t_{n}\right)\right]$ satisfying

$$
0<\int_{t_{1}}^{t_{n}} G_{n}(t, s) r(s) \Delta s<\infty
$$

for $t \in\left[\sigma\left(t_{1}\right), \sigma^{n-1}\left(t_{n}\right)\right]$.

(H2) $f:\left[t_{1}, \rho\left(t_{n}\right)\right] \times \mathbb{R} \rightarrow \mathbb{R}$ is such that $f(t, x) \geq 0$ for $x \in \mathbb{R}^{+}$and continuous with respect to $x$, where $\mathbb{R}^{+}$denotes the set of nonnegative real numbers.

Let us set

$$
M_{n}:=\max G_{n}(t, s) r(s), \quad m_{n}:=\min G_{n}(t, s) r(s)
$$

for $s \in\left[t_{1}, \rho\left(t_{n}\right)\right], t \in\left[\sigma\left(t_{1}\right), \sigma^{n-1}\left(t_{n}\right)\right]$, and

$$
A_{1_{n}}:=\max _{t \in\left[\sigma\left(t_{1}\right), \sigma^{n-1}\left(t_{n}\right)\right]} \int_{t_{1}}^{t_{n}} G_{n}(t, s) r(s) \Delta s, \quad A_{2_{n}}:=\min _{t \in\left[\sigma\left(t_{1}\right), \sigma^{n-1}\left(t_{n}\right)\right]} \int_{t_{1}}^{t_{n}} G_{n}(t, s) r(s) \Delta s .
$$

We refer to $[7,12]$ for a discussion of the fixed point index that we use below. In particular, we will make frequent use of the following lemma.

Lemma 2.4. Let $\mathscr{B}$ be a Banach space, and let $\mathscr{P} \subset \mathscr{B}$ be a cone in $\mathscr{B}$. Assume $r>0$ and that $\Psi: \mathscr{P}_{r} \rightarrow \mathscr{P}$ is compact operator such that $\Psi x \neq x$ for $x \in \partial \mathscr{P}_{r}:=\{x \in \mathscr{P}:\|x\|=r\}$. Then the following assertions hold.

(i) If $\|x\| \leq\|\Psi x\|$ for all $x \in \partial \mathscr{P}_{r}$, then $i\left(\Psi, \mathscr{P}_{r}, \mathscr{P}\right)=0$.

(ii) If $\|x\| \geq\|\Psi x\|$ for all $x \in \partial \mathscr{P}_{r}$, then $i\left(\Psi, \mathscr{P}_{r}, P\right)=1$.

Thus, if there exists $r_{1}>r_{2}>0$ such that condition (i) holds for $x \in \partial \mathscr{P}_{r_{1}}$ and (ii) holds for $x \in \partial \mathscr{P}_{r_{2}}$ (or (ii) and (i)), then, from the additivity properties of the index, we know that

$$
i\left(\Psi, \mathscr{P}_{r_{1}}, \mathscr{P}\right)=i\left(\Psi, \mathscr{P}_{r_{1}} \backslash \operatorname{int}\left(\mathscr{P}_{r_{2}}\right), \mathscr{P}\right)+i\left(\Psi, \mathscr{P}_{r_{2}}, \mathscr{P}\right)
$$

As a consequence of $i\left(\Psi, \mathscr{P}_{r_{1}} \backslash \operatorname{int}\left(\mathscr{P}_{r_{2}}\right), \mathscr{P}\right) \neq 0, \Psi$ has a fixed point (nonzero) whose norm is between $r_{1}$ and $r_{2}$.

Consider the Banach space of continuous functions on $\left[t_{1}, \sigma^{n-1}\left(t_{n}\right)\right]$ with the norm

$$
\|x\|=\max \left\{|x(t)|, t \in\left[\sigma\left(t_{1}\right), \sigma^{n-1}\left(t_{n}\right)\right]\right\},
$$


6 A right-focal boundary value problem on time scales

and cone $\mathscr{P}$ in $\mathscr{B}$ given by

$$
\mathscr{P}=\left\{x \in \mathscr{B}: x(t) \geq 0, t \in\left[\sigma\left(t_{1}\right), \sigma^{n-1}\left(t_{n}\right)\right], \min _{t \in\left[\sigma\left(t_{1}\right), \sigma^{n-1}\left(t_{n}\right)\right]} x(t) \geq \frac{m_{n}}{M_{n}}\|x\|\right\} .
$$

By Theorem 2.1, solving the BVP (1.1)-(1.2) is equivalent to solving the following integral equation in $\mathscr{P}$ :

$$
x(t)=\lambda \int_{t_{1}}^{t_{n}} G_{n}(t, s) r(s) f\left(s, x^{\sigma}(s)\right) \Delta s, \quad t \in\left[t_{1}, \sigma^{n-1}\left(t_{n}\right)\right],
$$

and consequently, it is equivalent to finding fixed points of the operator $\Psi_{n_{\lambda}}: \mathscr{B} \rightarrow \mathscr{B}$ defined by

$$
\Psi_{n_{\lambda}} x(t):=\lambda \int_{t_{1}}^{t_{n}} G_{n}(t, s) r(s) f\left(s, x^{\sigma}(s)\right) \Delta s, \quad t \in\left[t_{1}, \sigma^{n-1}\left(t_{n}\right)\right] .
$$

First, we prove that for every $\lambda>0$ given, this operator maps the cone $\mathscr{P}$ in itself.

Lemma 2.5. Let $\lambda>0$ be given. Under the hypotheses (H1) and (H2), the operator $\Psi_{n_{\lambda}}$ is a compact operator such that $\Psi_{n_{\lambda}}(\mathscr{P}) \subset \mathscr{P}$.

Proof. That $\Psi_{n_{\lambda}}$ is a compact operator follows by Arzela-Ascoli's theorem. Next, for all $x \in \mathscr{P}$, by $(\mathrm{H} 1),(\mathrm{H} 2)$, and the positivity property of the Green function, we have from (2.20), $\Psi_{n_{\lambda}} x(t) \geq 0$ for all $t \in\left[\sigma\left(t_{1}\right), \sigma^{n-1}\left(t_{n}\right)\right]$. If $x \in \mathscr{P}$, then

$$
\begin{aligned}
\min _{t \in\left[\sigma\left(t_{1}\right), \sigma^{n-1}\left(t_{n}\right)\right]} \Psi_{n_{\lambda}} x(t) & \geq \lambda m_{n} \int_{t_{1}}^{t_{n}} f\left(s, x^{\sigma}(s)\right) \Delta s \\
& \geq \lambda \frac{m_{n}}{M_{n}} \int_{t_{1}}^{t_{n}}\left\{\max _{t \in\left[\sigma\left(t_{1}\right), \sigma^{n-1}\left(t_{n}\right)\right]} G_{n}(t, s) r(s)\right\} f\left(s, x^{\sigma}(s)\right) \Delta s \\
& \geq \lambda \frac{m_{n}}{M_{n}} \max _{t \in\left[\sigma\left(t_{1}\right), \sigma^{n-1}\left(t_{n}\right)\right]} \int_{t_{1}}^{t_{n}} G_{n}(t, s) r(s) f\left(s, x^{\sigma}(s)\right) \Delta s \\
& =\frac{m_{n}}{M_{n}}\left\|\Psi_{n_{\lambda}} x\right\| .
\end{aligned}
$$

Therefore, $\Psi_{n_{\lambda}} x \in \mathscr{P}$.

\section{Noneigenvalue problem}

In this section we study the existence of at least two positive solutions to the following BVP:

$$
\begin{aligned}
(-1)^{n-1} x^{\Delta^{n}}(t) & =r(t) f\left(t, x^{\sigma}(t)\right) \quad \forall t \in\left[t_{1}, \rho\left(t_{n}\right)\right], \\
x\left(t_{1}\right) & =x^{\Delta}\left(t_{2}\right)=\cdots=x^{\Delta^{n-1}}\left(t_{n}\right)=0,
\end{aligned}
$$

which is problem (1.1)-(1.2) with $\lambda=1$. As an application, we also give an example to demonstrate our result.

Theorem 3.1. The boundary value problem (3.1) has at least two positive solutions, $x_{1}$ and $x_{2}$, if (H1) and (H2) are satisfied and, in addition, both of the following hold. 
(H3) There exists $0<k<R<+\infty$ such that

$$
f(t, x)>\frac{M_{n}}{m_{n}^{2}\left(t_{n}-t_{1}\right)} x \quad \forall x \in[0, k] \bigcup[R,+\infty], t \in\left[t_{1}, \rho\left(t_{n}\right)\right] .
$$

(H4) There exists $p>0$ such that

$$
f(t, x)<\frac{p}{M_{n}\left(t_{n}-t_{1}\right)} \quad \forall x \in[0, p], t \in\left[t_{1}, \rho\left(t_{n}\right)\right]
$$

where $M_{n}$ and $m_{n}$ are given as (2.14). Moreover, $0<\left\|x_{1}\right\|<p<\left\|x_{2}\right\|$.

Proof. Let $x \in \partial \mathscr{P}_{k}$. From condition (H3), we have

$$
\begin{aligned}
\left\|A_{1_{n}}\right\| & =\max _{t \in\left[\sigma\left(t_{1}\right), \sigma^{n-1}\left(t_{n}\right)\right]} \int_{t_{1}}^{t_{n}} G_{n}(t, s) r(s) f\left(s, x^{\sigma}(s)\right) \Delta s \\
& >m_{n} \frac{M_{n}}{m_{n}^{2}\left(t_{n}-t_{1}\right)} \int_{t_{1}}^{t_{n}} x^{\sigma}(s) \Delta s \\
& \geq \frac{M_{n}}{m_{n}\left(t_{n}-t_{1}\right)} \frac{m_{n}}{M_{n}}\|x\| \int_{t_{1}}^{t_{n}} \Delta s=\|x\| .
\end{aligned}
$$

If $x \in \partial \mathscr{P}_{R_{1}}, R_{1} \geq M_{n} / m_{n} R$, we have

$$
\min _{t \in\left[\sigma\left(t_{1}\right), \sigma^{n-1}\left(t_{n}\right)\right]} x(t) \geq \frac{m_{n}}{M_{n}}\|x\|=\frac{m_{n}}{M_{n}} R_{1} \geq R .
$$

Hence $x(s) \geq R$ for all $s \in\left[t_{1}, \rho\left(t_{n}\right)\right]$. Therefore using condition (H3) again, we arrive at the same conclusion.

Now, from (H4), if $\|x\|=p$,

$$
\begin{aligned}
\left\|A_{1_{n}}\right\| & =\max _{t \in\left[\sigma\left(t_{1}\right), \sigma^{n-1}\left(t_{n}\right)\right]} \int_{t_{1}}^{t_{n}} G_{n}(t, s) r(s) f\left(s, x^{\sigma}(s)\right) \Delta s \\
& \leq \int_{t_{1}}^{t_{n}} M_{n} f\left(s, x^{\sigma}(s)\right) \Delta s<\|x\| .
\end{aligned}
$$

Since we can choose $k>0$ small enough and $R_{1}$ sufficiently large so that $k<p<R_{1}$, we assure the existence of two solutions: $x_{1} \in \mathscr{P}_{p} \backslash \operatorname{int}\left(\mathscr{P}_{k}\right)$ and $x_{2} \in \mathscr{P}_{R_{1}} \backslash \operatorname{int}\left(\mathscr{P}_{p}\right)$.

Example 3.2. We illustrate Theorem 3.1 with specific time scale

$$
\mathbf{T}=\mathbf{T}_{c}=\left\{c^{m}: m \in \mathbb{Z}\right\} \cup\{0\},
$$

where $c>1$ and the following specific parameter values for $n=3$. Let $c=11 / 10, t_{1}=1$, $t_{2}=(11 / 10)^{3}$, and $t_{3}=(11 / 10)^{4}$. Bohner and Peterson [6] show that

$$
h_{j}(t, s)=\prod_{\nu=0}^{j-1} \frac{t-c^{\nu} s}{\sum_{\mu=0}^{\nu} c^{\mu}}
$$


8 A right-focal boundary value problem on time scales

for all $s, t \in \mathbf{T}$. Using this formula, we have

$$
\begin{aligned}
& G_{3}(t, s)
\end{aligned}
$$

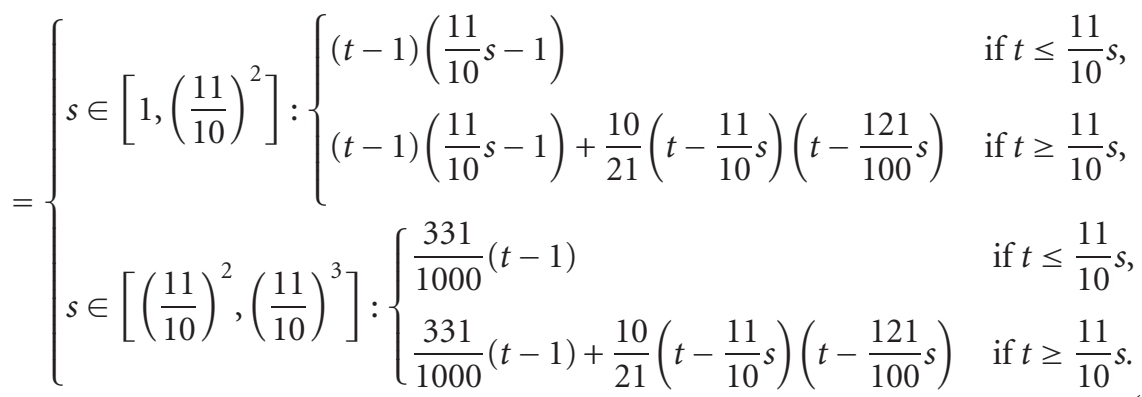

If $r(s)=s$, then $m_{3}=\min G(t, s) r(s)=10^{-2}, M_{3}=\max G(t, s) r(s)=3870659646821 \cdot 10^{-13}$ for $t \in\left[11 / 10,(11 / 10)^{6}\right], s \in\left[1,(11 / 10)^{3}\right]$.

Let $k=1 / 18000, p=1 / 5, R=2 / 5$, let

$$
f(t, x)= \begin{cases}2.10^{4} k \sin \frac{t \pi}{6} & \text { if } x \in[0, k], \\ L(x) \sin \frac{t \pi}{6} & \text { if } x \in[k, p], \\ K(x) \sin \frac{t \pi}{6} & \text { if } x \in[p, R], \\ 2.10^{4} \frac{k+1}{R} x \sin \frac{t \pi}{6} & \text { if } x \in[R,+\infty),\end{cases}
$$

where

$$
\begin{gathered}
L(x)=1+\frac{p-x}{p-k}\left[2.10^{4} k-1\right], \\
K(x)=1+\frac{p-x}{p-R}\left[2.10^{4}(k+1)-1\right] .
\end{gathered}
$$

Note that $f$ is continuous and nonnegative valued for $x \geq 0$.

For $t \in\left[1,(11 / 10)^{3}\right]$ and $x \in[0, k] \cup[R, \infty), f(t, x)>(8340,14144, \ldots) x$. Indeed; for $x \in[0, k], f(t, x)=2.10^{4} k \sin (t \pi / 6) \geq 10^{4} k>(8340,14144, \ldots) x$, for $x \in[R, \infty), f(t, x)=$ $2.10^{4}((k+1) / R) x \sin (t \pi / 6) \geq 10^{4}((k+1) / R) x=(900050 / 36) x>(8340,14144, \ldots) x$. So (H3) is verified.

For $x \in[0, k], f(t, x)=2.10^{4} k \sin (t \pi / 6)<2.10^{4} k<p / 0,17963731420896261$.

For $x \in[k, p], f(t, x)=1+((p-x) /(p-k))\left[2.10^{4} k-1\right] \sin (t \pi / 6) \leq 2.10^{4} k \sin (t \pi / 6)<$ $2.10^{4} k<p / 0,17963731420896261$. Hence it verifies the (H4).

We conclude from Theorem 3.1 that for these parameter values, (3.1) for $n=3$ has at least two positive solutions, $x_{1}$ and $x_{2}$ such that $0<\left\|x_{1}\right\|<1 / 5<\left\|x_{2}\right\|$. 


\section{Eigenvalue problem}

Define the nonnegative extended real numbers $f_{0}, f^{0}, f_{\infty}$, and $f^{\infty}$ by

$$
\begin{aligned}
f_{0}:=\liminf _{x \rightarrow 0^{+}} \min _{t \in\left[t_{1}, \rho\left(t_{n}\right)\right]} \frac{f(t, x)}{x}, & f^{0}:=\limsup _{x \rightarrow 0^{+}} \max _{t \in\left[t_{1}, \rho\left(t_{n}\right)\right]} \frac{f(t, x)}{x}, \\
f_{\infty}:=\liminf _{x \rightarrow \infty} \min _{t \in\left[t_{1}, \rho\left(t_{n}\right)\right]} \frac{f(t, x)}{x}, & f^{\infty}:=\limsup _{x \rightarrow \infty} \max _{t \in\left[t_{1}, \rho\left(t_{n}\right)\right]} \frac{f(t, x)}{x},
\end{aligned}
$$

respectively.

These numbers can be regarded as generalized super or sublinear conditions on the function $f(t, x)$ at $x=0$ and $x=\infty$. Thus, if $f_{0}=f^{0}=0(+\infty)$, then $f(t, x)$ is superlinear (sublinear) at $x=0$ and if $f_{\infty}=f^{\infty}=0(+\infty)$, then $f(t, x)$ is sublinear (superlinear) at $x=+\infty$.

First, we obtain an existence result for $\lambda$ belonging to a given interval.

TheOREM 4.1. If (H1)-(H2) hold and either

(a) $M_{n} /\left(m_{n} A_{2_{n} f_{0}}\right)<\lambda<1 /\left(A_{1_{n}} f^{\infty}\right)$, or

(b) $M_{n} /\left(m_{n} A_{2_{n}} f_{\infty}\right)<\lambda<1 /\left(A_{1_{n}} f^{0}\right)$

is satisfied, where $M_{n}, m_{n}, A_{1_{n}}$, and $A_{2_{n}}$ are given as in (2.14) and (2.15), then the eigenvalue problem (1.1)-(1.2) has at least one positive solution.

Proof. Assume $(a)$ holds. First we consider $f_{0}<\infty$. Since

$$
\frac{M_{n}}{m_{n} A_{2_{n}} f_{0}}<\lambda
$$

there is an $\epsilon>0$ so that

$$
\lambda\left(f_{0}-\epsilon\right) \frac{m_{n}}{M_{n}} A_{2_{n}} \geq 1
$$

Using the definition of $f_{0}$, there is an $r_{1}>0$, sufficiently small, so that

$$
f_{0}-\epsilon<\min _{t \in\left[t_{1}, \rho\left(t_{n}\right)\right]} \frac{f(t, x)}{x}
$$

for $0<x \leq r_{1}$.

It follows that $f(t, x)>\left(f_{0}-\epsilon\right) x$ for $0<x \leq r_{1}, t \in\left[t_{1}, \rho\left(t_{n}\right)\right]$.

Assume that $x \in \partial \mathscr{P}_{r_{1}}$, then

$$
\begin{aligned}
\Psi_{n_{\lambda}} x(t) & =\lambda \int_{t_{1}}^{t_{n}} G_{n}(t, s) r(s) f\left(s, x^{\sigma}(s)\right) \Delta s \\
& >\lambda\left(f_{0}-\epsilon\right) \int_{t_{1}}^{t_{n}} G_{n}(t, s) r(s) x^{\sigma}(s) \Delta s \\
& \geq \lambda\left(f_{0}-\epsilon\right) \frac{m_{n}}{M_{n}}\|x\| A_{2_{n}} \geq\|x\| .
\end{aligned}
$$


Next, we consider the case $f_{0}=\infty$. Choose $K>0$ sufficiently large so that

$$
\lambda K \frac{m_{n}}{M_{n}} A_{2_{n}} \geq 1
$$

for any $t \in\left[t_{1}, \sigma^{n-1}\left(t_{n}\right)\right]$.

So there exists $r_{1}>0$ so that $f(t, x)>K x$ for $0<x \leq r_{1}$.

Assume that $x \in \partial \mathscr{P}_{r_{1}}$, then

$$
\Psi_{n_{\lambda}} x(t)>\lambda K \int_{t_{1}}^{t_{n}} G_{n}(t, s) r(s) x^{\sigma}(s) \Delta s \geq \lambda K \frac{m_{n}}{M_{n}}\|x\| A_{2_{n}} \geq\|x\| .
$$

Finally, we use the assumption

$$
\lambda<\frac{1}{A_{1_{n}} f^{\infty}} .
$$

Pick an $\epsilon_{1}>0$ so that

$$
\lambda\left(f^{\infty}+\epsilon_{1}\right) A_{1_{n}} \leq 1
$$

Using the definition of $f^{\infty}$, there is an $r>r_{1}$ sufficiently large, so that

$$
\max _{t \in\left[t_{1}, \rho\left(t_{n}\right)\right]} \frac{f(t, x)}{x}<f^{\infty}+\epsilon_{1},
$$

for $x \geq r$.

It follows that $f(t, x)<\left(f^{\infty}+\epsilon_{1}\right) x$ for $x \geq r$.

We now show that there is an $r_{2} \geq r$ such that if $x \in \partial \mathscr{P}_{r_{2}}$, then $\left\|\Psi_{n_{\lambda}} x\right\|<\|x\|$.

Pick $r_{2} \geq r M_{n} / m_{n}>r_{1}$. Now assume $x \in \partial \mathscr{P}_{r_{2}}$ and consider

$$
\Psi_{n_{\lambda}} x(t)<\lambda\left(f^{\infty}+\epsilon_{1}\right) \int_{t_{1}}^{t_{n}} G_{n}(t, s) r(s) x^{\sigma}(s) \Delta s \leq \lambda\left(f^{\infty}+\epsilon_{1}\right) A_{1_{n}}\|x\| \leq\|x\| .
$$

Therefore, by Lemma 2.4, $\Psi_{n_{\lambda}}$ has a fixed point $x$ with $r_{1}<\|x\|<r_{2}$. This shows that condition (a) yields the existence of a positive solution of the eigenvalue problem (1.1)(1.2). This completes the proof of the theorem.

The proof of part $(b)$ is similar.

Our next results give criteria for the existence of one, more than one, or no positive solutions of the eigenvalue problem (1.1)-(1.2) in terms of the superlinear or sublinear behavior of $f(t, x)$. For the next three theorems, in addition to the assumptions (H1) and (H2) we assume.

(H5) $f(t, x)>0$ on $\left[t_{1}, \rho\left(t_{n}\right)\right] \times \mathbb{R}^{+}$.

THEOREM 4.2. If hypotheses (H1), (H2), and (H5) are satisfied, then the following assertions hold.

(a) If $f_{0}=\infty$ or $f_{\infty}=\infty$, then there is a $\lambda_{0}>0$ such that for all $0<\lambda \leq \lambda_{0}$ the eigenvalue problem (1.1)-(1.2) has a positive solution. 
(b) If $f^{0}=0$ or $f^{\infty}=0$, then there is a $\lambda_{0}>0$ such that for all $\lambda \geq \lambda_{0}$ the eigenvalue problem (1.1)-(1.2) has a positive solution.

Proof of part (a). Let $r>0$ be given. From conditions (H2) and (H5) we can define

$$
L:=\max \left\{f(t, x):(t, x) \in\left[t_{1}, \rho\left(t_{n}\right)\right] \times[0, r]\right\}>0 .
$$

Then if $x \in \partial \mathscr{P}_{r}$, it follows that

$$
\Psi_{n_{\lambda}} x(t) \leq \lambda L \int_{t_{1}}^{t_{n}} G_{n}(t, s) r(s) \Delta s \leq \lambda L A_{1_{n}} .
$$

It follows that we can pick $\lambda_{0}>0$ sufficiently small so that for all $0<\lambda \leq \lambda_{0}$,

$$
\left\|\Psi_{n_{\lambda}} x\right\| \leq\|x\|
$$

for all $x \in \partial \mathscr{P}_{r}$.

Fix $\lambda \leq \lambda_{0}$. Choose $T>0$ sufficiently large so that

$$
\lambda \frac{m_{n}}{M_{n}} T A_{2_{n}} \geq 1
$$

Since $f_{0}=\infty$, there exists $s<r$ such that

$$
\min _{t \in\left[t_{1}, \rho\left(t_{n}\right)\right]} \frac{f(t, x)}{x}>T
$$

for $0<x \leq s$. Hence, we have that

$$
f(t, x)>T x \quad \text { for } t \in\left[t_{1}, \rho\left(t_{n}\right)\right], 0<x \leq s .
$$

Now, let $x \in \partial \mathscr{P}_{s}$. In this case,

$$
\Psi_{n_{\lambda}} x(t)>\lambda T \int_{t_{1}}^{t_{n}} G_{n}(t, s) r(s) x^{\sigma}(s) \Delta s \geq \lambda T \frac{m_{n}}{M_{n}}\|x\| A_{2_{n}} \geq\|x\|
$$

for $t \in\left[\sigma\left(t_{1}\right), \sigma^{n-1}\left(t_{n}\right)\right]$. Hence we have shown that if $x \in \partial \mathscr{P}_{s}$, then $\left\|\Psi_{n_{\lambda}} x\right\| \geq\|x\|$.

It follows from Lemma 2.4 that the operator $\Psi_{n_{\lambda}}$ has a fixed point.

When $f_{\infty}=\infty$, there is a $w>r$ such that

$$
\min _{t \in\left[t_{1}, \rho\left(t_{n}\right)\right]} \frac{f(t, x)}{x}>T
$$

for $x \geq \omega$.

It follows that $f(t, x)>T x$ for $t \in\left[t_{1}, \rho\left(t_{n}\right)\right], x \geq \omega$.

Let $\omega_{0}:=\omega M_{n} / m_{n}$. Next if $x \in \partial \mathscr{P}_{\omega_{0}}$, then we show that $\left\|\Psi_{n_{\lambda}} x\right\|>\|x\|$. In fact,

$$
\Psi_{n_{\lambda}} x(t)>\lambda T \int_{t_{1}}^{t_{n}} G_{n}(t, s) r(s) x^{\sigma}(s) \Delta s \geq \lambda T \frac{m_{n}}{M_{n}}\|x\| A_{2_{n}} \geq\|x\|
$$

for $t \in\left[t_{1}, \sigma^{n-1}\left(t_{n}\right)\right]$. This completes the proof of part $(a)$. 
Part (b) holds in an analogous way.

Similar to the proof of Theorem 4.2, we get the next result.

TheOREM 4.3. Under the hypotheses of Theorem 4.2, the following assertions hold.

(a) If $f_{0}=f_{\infty}=\infty$, then there is a $\lambda_{0}>0$ such that for all $0<\lambda \leq \lambda_{0}$, the eigenvalue problem (1.1)-(1.2) has two positive solutions.

(b) If $f^{0}=f^{\infty}=0$, then there is a $\lambda_{0}>0$ such that for all $\lambda \geq \lambda_{0}$, the eigenvalue problem (1.1)-(1.2) has two positive solutions.

Now, we give a nonexistence result as follows.

THEOREM 4.4. Under the hypotheses of Theorem 4.2, the following assertions hold.

(a) If there is a constant $c>0$ such that $f(t, x) \geq c x$ for $x \geq 0$, then there is a $\lambda_{0}>0$ such that the eigenvalue problem (1.1)-(1.2) has no positive solutions for $\lambda \geq \lambda_{0}$.

(b) If there is a constant $c>0$ such that $f(t, x) \leq c x$ for $x \geq 0$, then there is a $\lambda_{0}>0$ such that the eigenvalue problem (1.1)-(1.2) has no positive solutions for $0<\lambda \leq \lambda_{0}$.

Proof of part (b). Assume there is constant $c>0$ such that $f(t, x) \leq c x$ for $x \geq 0$. Assume $x(t)$ is a positive solution of the eigenvalue problem (1.1)-(1.2). We will show that for $\lambda$ sufficiently small and positive that this leads to a contradiction. Since $\Psi_{n_{\lambda}} x(t)=x(t)$ for $t \in\left[t_{1}, \sigma^{n-1}\left(t_{n}\right)\right]$

$$
\begin{aligned}
x(t) & =\lambda \int_{t_{1}}^{t_{n}} G_{n}(t, s) r(s) f\left(s, x^{\sigma}(s)\right) \Delta s \leq c \lambda \int_{t_{1}}^{t_{n}} G_{n}(t, s) r(s) x^{\sigma}(s) \Delta s \\
& \leq c \lambda\|x\| \int_{t_{1}}^{t_{n}} G_{n}(t, s) r(s) \Delta s \leq c \lambda A_{1_{n}}\|x\|
\end{aligned}
$$

for $t \in\left[t_{1}, \sigma^{n-1}\left(t_{n}\right)\right]$. Pick $\lambda_{0}$ sufficiently small so that for $0<\lambda \leq \lambda_{0}$,

$$
c \lambda A_{1_{n}}<1,
$$

then we have $x(t)<\|x\|$ for $t \in\left[t_{1}, \sigma^{n-1}\left(t_{n}\right)\right]$ which is a contradiction.

The proof of part $(a)$ is similar.

\section{References}

[1] R. P. Agarwal and M. Bohner, Basic calculus on time scales and some of its applications, Results in Mathematics 35 (1999), no. 1-2, 3-22.

[2] D. R. Anderson, Positivity of Green's function for an n-point right focal boundary value problem on measure chains, Mathematical and Computer Modelling 31 (2000), no. 6-7, 29-50.

[3] — Discrete third-order three-point right-focal boundary value problems, Computers \& Mathematics with Applications 45 (2003), no. 6-9, 861-871.

[4] B. Aulbach, Analysis auf Zeitmengen, Lecture Notes, Universitat Augsburg, Augsburg, 1990.

[5] K. L. Boey and P. J. Y. Wong, Two-point right focal eigenvalue problems on time scales, Applied Mathematics and Computation 167 (2005), no. 2, 1281-1303.

[6] M. Bohner and A. Peterson, Dynamic Equations on Time Scales. An Introduction with Applications, Birkhäuser Boston, Massachusetts, 2001.

[7] K. Deimling, Nonlinear Functional Analysis, Springer, Berlin, 1985.

[8] L. Erbe and A. Peterson, Eigenvalue conditions and positive solutions, Journal of Difference Equations and Applications 6 (2000), no. 2, 165-191. 
[9] __ Positive solutions for a nonlinear differential equation on a measure chain, Mathematical and Computer Modelling 32 (2000), no. 5-6, 571-585.

[10] L. Erbe, A. Peterson, and R. Mathsen, Existence, multiplicity, and nonexistence of positive solutions to a differential equation on a measure chain, Journal of Computational and Applied Mathematics 113 (2000), no. 1-2, 365-380.

[11] S. Hilger, Analysis on measure chains - a unified approach to continuous and discrete calculus, Results in Mathematics 18 (1990), no. 1-2, 18-56.

[12] M. A. Krasnosel'skiĭ, Positive Solutions of Operator Equations, Groningen and P. Noordhoff, The Netherlands, 1964.

[13] F. Merdivenci Atici, A. Cabada, and V. Otero-Espinar, Criteria for existence and nonexistence of positive solutions to a discrete periodic boundary value problem, Journal of Difference Equations and Applications 9 (2003), no. 9, 765-775.

[14] F. Merdivenci Atici and G. Sh. Guseinov, On the existence of positive solutions for nonlinear differential equations with periodic boundary conditions, Journal of Computational and Applied Mathematics 132 (2001), no. 2, 341-356.

Ilkay Yaslan Karaca: Department of Mathematics, Ege University, 35100 Bornova, Izmir, Turkey

E-mail address: ilkay.karaca@ege.edu.tr 